\title{
ARTICLE OPEN \\ Computer predictions on Rh-based double perovskites with unusual electronic and magnetic properties
}

\author{
Anita Halder ${ }^{1}$, Dhani Nafday ${ }^{1}$, Prabuddha Sanyal ${ }^{2}$ and Tanusri Saha-Dasgupta ${ }^{1,3}$
}

In search for new magnetic materials, we make computer prediction of structural, electronic and magnetic properties of yet-to-be synthesized $\mathrm{Rh}$-based double perovskite compounds, $\mathrm{Sr}(\mathrm{Ca})_{2} \mathrm{BRhO}_{6}(\mathrm{~B}=\mathrm{Cr}, \mathrm{Mn}, \mathrm{Fe})$. We use combination of evolutionary algorithm, density functional theory, and statistical-mechanical tool for this purpose. We find that the unusual valence of $\mathrm{Rh}^{5+}$ may be stabilized in these compounds through formation of oxygen ligand hole. Interestingly, while the $\mathrm{Cr}-\mathrm{Rh}$ and $\mathrm{Mn}-\mathrm{Rh}$ compounds are predicted to be ferromagnetic half-metals, the Fe-Rh compounds are found to be rare examples of antiferromagnetic and metallic transition-metal oxide with three-dimensional electronic structure. The computed magnetic transition temperatures of the predicted compounds, obtained from finite temperature Monte Carlo study of the first principles-derived model Hamiltonian, are found to be reasonably high. The prediction of favorable growth condition of the compounds, reported in our study, obtained through extensive thermodynamic analysis should be useful for future synthesize of this interesting class of materials with intriguing properties.

npj Quantum Materials (2018)3:17; doi:10.1038/s41535-018-0091-6

\section{INTRODUCTION}

While magnetic materials are technologically important and useful in every day life, they are rare. Only about $5 \%$ of known inorganic compounds are found to be magnetic (see link http://pratt.duke. edu/news/predicting-magnets). Understandably, experimental effort in discovering new magnetic materials is limited by the high cost and time-consuming procedure of synthesis. A natural alternative is the computational modeling of materials, which has emerged as a powerful tool for predicting materials with desired properties. $^{1-3}$

In this study we will focus on the computer prediction of new double perovskite (DP) compounds, which pose the promise of exhibiting novel electronic and magnetic properties. These compounds have a general formula of $\mathrm{A}_{2} \mathrm{BB}^{\prime} \mathrm{O}_{6}$, where $A$ site is occupied by alkaline-earth or rare-earth ions, and $B$ and $B^{\prime}$ sites are occupied by transition metal (TM) elements. Some of the magnetic members of this family are well-known for their spectacularly high magnetic transition temperature, like $\mathrm{Sr}_{2} \mathrm{FeMoO}_{6}{ }^{4} \mathrm{Sr}_{2} \mathrm{CrMoO}_{6}{ }^{5,6}$ $\mathrm{Sr}_{2} \mathrm{CrWO}_{6}{ }^{7} \mathrm{Sr}_{2} \mathrm{CrReO}_{6}{ }^{8}$ or $\mathrm{Sr}_{2} \mathrm{CrOsO}_{6} .{ }^{9}$ Due to the presence of two TM elements ( $B$ and $B^{\prime}$ ) in DP, instead of one $B$ element as in simple perovskites, DPs provide large flexibility in choice of different combinations of $B$ and $B^{\prime}$, leading to possible engineering of novel magnetic properties. In spite of the fact that about a thousand of DP compounds have already been synthesized, this forms only a small percentage of all the possible compounds that may be synthesized (see Fig. 2 in ref. ${ }^{10}$ ). Therefore, possible predictions can be made on a large number of new DP compounds, that has remained unexplored so far.

In the present work, in particular, we restrict our search to $3 d-4 d$ DPs with $3 d$ TM cation at B site, $\mathrm{B}^{\prime}$ site being occupied by $4 d$ TM element, Rh. As most of the DP compounds found in literature
( 700 in number) have a divalent cation at $A$ site, we consider compounds having $\mathrm{Sr}^{2+}$ or $\mathrm{Ca}^{2+}$ at A site. For B site element, we consider $\mathrm{Cr}, \mathrm{Mn}$ and $\mathrm{Fe}$, which are neighboring elements in $3 d \mathrm{TM}$ series. This allows us to study the trend in properties upon increasing filling. Based on our first-principles density functional theory (DFT) calculations combined with genetic evolutionary algorithm and finite temperature study of model Hamiltonian, we make predictions of structure and properties of $\mathrm{Sr}_{2} \mathrm{BRhO}_{6}$ and $\mathrm{Ca}_{2} \mathrm{BRhO}_{6}(\mathrm{~B}=\mathrm{Cr}, \mathrm{Mn}, \mathrm{Fe})$ compounds. Note that there are only a few Rh-based DPs known. While among the $\mathrm{Ca}^{2+}$-based compounds, there is none known, among the $\mathrm{Sr}^{2+}$-based compounds, the known compounds are $\mathrm{Sr}_{2} \mathrm{NbRhO}_{6}{ }^{11}$ $\mathrm{Sr}_{2} \mathrm{TaRhO}_{6},{ }^{11} \mathrm{Sr}_{2} \mathrm{SbRhO}_{6},{ }^{12}$ All these compounds are nonmagnetic ${ }^{13}$ with $d^{0}$ and low-spin $d^{6}$ valences at $B$ and Rh sites, respectively. On the contrary, our study showed all the predicted Rh DPs to be magnetic with reasonably high magnetic transition temperature. The $\mathrm{Cr}-\mathrm{Rh}, \mathrm{Mn}-\mathrm{Rh} \mathrm{DPs}$ are found to be half-metallic ferromagnets, making them suitable for spintronics application. ${ }^{14}$ Fe-Rh compounds, on the other hand, are found to be in the curious state of metallic, antiferromagnetism, adding these compounds in the list of antiferromagnetic (AFM) metallic oxides with three-dimensional electronic structure, like $\mathrm{CaCrO} 3 .{ }^{15}$ We also estimated the growth conditions of these compounds in terms of temperature and partial pressure of reactants from extensive thermodynamic stability analysis. ${ }^{16,17}$ This indicated that high temperature, or low oxygen partial pressure may be required for synthesizing the predicted compounds.

\section{RESULTS}

In the results and discussions section, we report (a) the theoretically predicted crystal structures of the compounds, (b)

\footnotetext{
${ }^{1}$ Department of Condensed Matter Physics and Material Sciences, S N Bose National Centre for Basic Sciences, JD Block, Sector III, Salt Lake, Kolkata 700106, India; ${ }^{2}$ Department of Physics, Indian Institute of Technology, Roorkee 247667, India and ${ }^{3}$ Center for Mathematical, Computational and Data Science, Indian Association for the Cultivation of Science. 2A\&B, Raja S.C. Mullick Rd, Jadavpur, Kolkata 700032, India

Correspondence: Tanusri Saha-Dasgupta (t.sahadasgupta@gmail.com)
}

Received: 20 November 2017 Revised: 15 February 2018 Accepted: 26 February 2018

Published online: 19 March 2018 


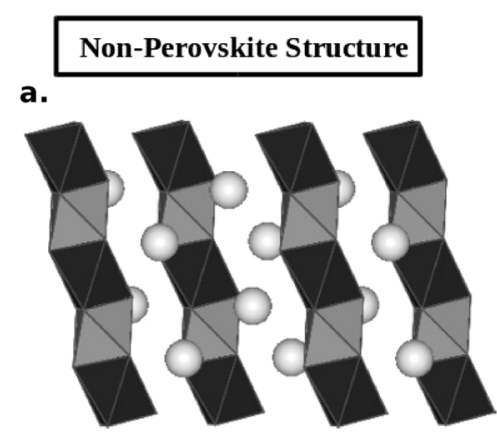

$\mathrm{C} 2 / \mathrm{m}$ (12)

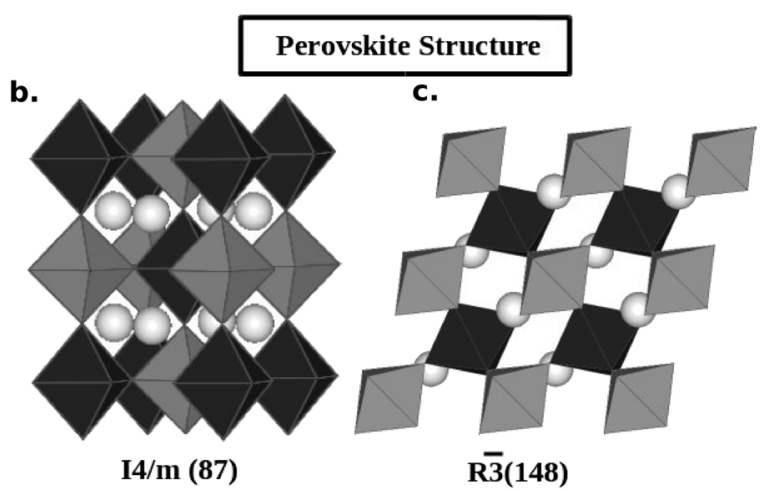

Fig. 1 Schematic representation of low-energy crystal structures of $\mathrm{Sr}_{2} \mathrm{BRhO}_{6}$ and $\mathrm{Ca}_{2} \mathrm{BRhO}_{6}(\mathrm{~B}=\mathrm{Cr}, \mathrm{Mn}$, $\mathrm{Fe})$ compounds, as given by the evolutionary search. a Non-Perovskite structure with $\mathrm{C} 2 / \mathrm{m}$ symmetry, b Perovskite structure with $14 / \mathrm{m}$ symmetry and $\mathbf{c}$ Perovskite structure with $\mathrm{R} \overline{3}$ symmetry. The $\mathrm{BO}_{6}$ and $\mathrm{RhO}_{6}$ polyhedra are shown as dark and light gray polyhedra, respectively, while the $\mathrm{Sr}^{2+}$ or $\mathrm{Ca}^{2+}$ ions are shown as balls

the electronic structure defined in terms of magnetic moments, assigned valence states and density of states (DOS), (c) the magnetic ground state, (d) the DFT-derived model Hamiltonian and calculated magnetic transition temperatures obtained through Monte Carlo study of model Hamiltonian, and (e) the predicted growth conditions of the compounds.

\section{Predicted crystal structures}

Compilation of crystal structure data of so-far synthesized DP compounds shows ${ }^{10}$ that while compounds with relatively smaller tolerance values, 0.93 or less, are predominantly of monoclinic $\mathrm{P} 2 / \mathrm{n}$ symmetry, compounds with relatively larger tolerance factors, 0.97 and beyond form in either cubic Fm-3m, or tetragonal $14 / \mathrm{m}$ or rhombohedral $\mathrm{R} \overline{3}$ symmetry. Our search based on evolutionary algorithm results into either $14 / \mathrm{m}$ or $\mathrm{R} \overline{3}$ symmetry of the compounds, in agreement with this general expectation. We note here that larger size of $\mathrm{Sr}^{2+}$ compared to $\mathrm{Ca}^{2+}$ drives the tolerance factor $t\left(t=\frac{r_{\mathrm{A}}+r_{0}}{\sqrt{2}\left(\frac{r_{\mathrm{B}}+r_{\mathrm{B}^{\prime}}}{2}+r_{0}\right)}\right.$ where $r_{\mathrm{A}}, r_{\mathrm{O}}, r_{\mathrm{B}^{\prime}}, r_{\mathrm{B}^{\prime}}$ are the ionic radii of $A, O, B$ and $B^{\prime}$ ions respectively) $>1$ (1.03-1.01) for $\mathrm{Sr}$ based compounds and $<1(0.98-0.97)$ for Ca-based compounds. $t$ $>1$ makes non-perovskite structures with face shared $\mathrm{BO}_{6}$ and $\mathrm{B}^{\prime} \mathrm{O}_{6}$ units as possible structures for Sr-based compounds, which compete with perovskite structures having corner-shared $\mathrm{BO}_{6}$ and $\mathrm{B}^{\prime} \mathrm{O}_{6}$ units. This poses a challenge to the evolutionary algorithm. While for all Ca-based compounds, low-energy structures are found to be of perovskite-based $R \overline{3}$ symmetry, in case of Sr-based compounds, a non-perovskite phase with $\mathrm{C} 2 / \mathrm{m}$ symmetry is found to compete with $\mathrm{R} \overline{3}$ for $\mathrm{Sr}_{2} \mathrm{CrRhO}_{6}$ and $\mathrm{Sr}_{2} \mathrm{FeRhO}_{6}$, and $14 / \mathrm{m}$ for $\mathrm{Sr}_{2} \mathrm{MnRhO}_{6}$. The predicted crystal structure data have been provided in Supplementary Information.

Figure 1 shows the schematic representation of $\mathrm{C} 2 / \mathrm{m}, 14 / \mathrm{m}$ and $\mathrm{R} \overline{3}$ structures. The crystal structure of non-perovskite monoclinic $\mathrm{C} 2 / \mathrm{m}$ symmetry can be derived from the hexagonal $P$ 3m1 symmetry by introduction of distortion, with their lattice constants connected by the transformation $a^{\prime}=-a+b, b^{\prime}=-a$ $-b$, and $c^{\prime}=c, a^{\prime}(a), b^{\prime}(b)$ and $c^{\prime}(c)$ being the lattice constants of the monoclinic (hexagonal) unit cell. The distorted $\mathrm{BO}_{6}$ and $\mathrm{RhO}_{6}$ octahedra in this structure, face-share and form chain-like structures with alternating $\mathrm{BO}_{6}$ and $\mathrm{RhO}_{6}$ units running along crystallographic c-direction, and $\mathrm{Sr}$ atoms positioned in between the chains. The $14 / \mathrm{m}$ symmetry of the perovskite-based structure shows $a^{0} a^{0} c^{-}$Glazer tilt pattern resulting into $180^{\circ} \mathrm{B}-\mathrm{O}-\mathrm{Rh}$ bond angles along the crystallographic $c$-axis and in-plane $\mathrm{B}-\mathrm{O}-\mathrm{Rh}$ bond angle bent from $180^{\circ}$. The $\mathrm{R} \overline{3}$ symmetry of the perovskitebased structure, on the other hand shows $a^{-} a^{-} a^{-}$Glazer tilt pattern resulting into $\mathrm{B}-\mathrm{O}-\mathrm{Rh}$ bond angles that are same between in-plane and out-of-plane directions, and having a value that deviates from $180^{\circ}$. The deviation of $\angle \mathrm{B}-\mathrm{O}-\mathrm{Rh}$ from $180^{\circ}$ for perovskite-structures of $\mathrm{Sr}_{2} \mathrm{BRhO}_{6}$ compounds is found to be around $20^{\circ}$, while for $\mathrm{Ca}_{2} \mathrm{BRhO}_{6}$ compounds this deviation is found to increase to about $30^{\circ}$. The $\mathrm{B}-\mathrm{O}$ and $\mathrm{Rh}-\mathrm{O}$ bond lengths show a definitive trend as a function of choice of Hubbard $U$ parameter on B site $\left(U_{B}\right)$. While $\mathrm{B}-\mathrm{O}$ bondlengths are found to be less than $2 \AA$ $(\approx 1.88-1.90 \AA)$ in DFT calculations within generalized gradient approximation (GGA), within GGA $+U$ calculations, for choice of $U_{\mathrm{B}}=4 \mathrm{eV}$ or more, the $\mathrm{B}-\mathrm{O}$ bond lengths are found to be more than $2 \AA(\approx 2.01-2.02 \AA)$. This occurs with a concomitant decrease of $\mathrm{Rh}-\mathrm{O}$ bond length from a value $2.03-2.04 \AA$ obtained within GGA calculation to a value $1.98-1.99 \AA$ in $\mathrm{GGA}+U$ calculations for $U_{B}=4 \mathrm{eV}$ or more. The observed $U_{B}$-dependent evolution of bond length has important implications in valences of $B$ cation and $\mathrm{Rh}$ cation, as will be discussed in the following.

Magnetic moment, valence states, electronic structure In order to discuss the electronic properties of $\mathrm{B}$ ion and $\mathrm{Rh}$ ion in $\mathrm{Sr}_{2} \mathrm{BRhO}_{6}$ and $\mathrm{Ca}_{2} \mathrm{BRhO}_{6}(\mathrm{~B}=\mathrm{Cr}, \mathrm{Mn}, \mathrm{Fe})$ compounds, we start with discussion of calculated magnetic moments, as given in GGA $+U$ calculations. As expected, the magnetic moments at $\mathrm{Sr}$ or $\mathrm{Ca}$ sites are found to be negligible small. We thus limit our discussion to $B$, $\mathrm{Rh}$ and $\mathrm{O}$ site magnetic moment. The first observation to be made is that, irrespective of the choice of $U_{B}$, the magnetic moment at $\mathrm{Rh}$ site, along with that at $\mathrm{O}$ sites are aligned in opposite direction to that at $B$ site $\left(M_{B}\right)$. The total moment in the formula unit is found to be 1,2 and $3 \mu_{\mathrm{B}}$ aligned in the direction of $M_{\mathrm{B}}$, for $\mathrm{Sr}$ $(\mathrm{Ca})_{2} \mathrm{CrRhO}_{6}, \mathrm{Sr}(\mathrm{Ca})_{2} \mathrm{MnRhO}_{6}$ and $\mathrm{Sr}(\mathrm{Ca})_{2} \mathrm{FeRhO}_{6}$, respectively. The values of atom-projected magnetic moments at $\mathrm{B}, \mathrm{Rh}$ and $\mathrm{O}$ sites, however, show interesting evolution as a function of $U_{B}$, as presented in Fig. 2. The precise value of the moment will understandably depend on the choice of sphere radii on which the projection is made. A comparison of magnetic moments calculated in linear muffin-tin orbital and plane wave basis is presented in Supplementary Information, Table. S2. Although the detailed values defer, the basic conclusion is found to hold good. For reference, Fig. 2 also includes the result of GGA calculation, shown as the data point corresponding to $U_{\mathrm{B}}=0 \mathrm{eV}$.

Note that the total B-cation oxidation state in DP formula unit of $\mathrm{Sr}(\mathrm{Ca})_{2} \mathrm{BRhO}_{6}$ is $8+$, considering the nominal valences of $2-$ for oxygen and $2+$ for $\mathrm{Sr} / \mathrm{Ca}$. This can be realized by a combination of $4+/ 4+, 3+/ 5+$, or $5+/ 3+$ valences of $\mathrm{B} / \mathrm{Rh}$, as the possible oxidation states of $\mathrm{Rh}$ in octahedral coordination can be $3+$ or $4+$ or $5+.{ }^{18}$ The observation of net moment of 1,2 and $3 \mu_{\mathrm{B}}$ aligned in the direction of $M_{\mathrm{B}}$, for $\mathrm{Sr}(\mathrm{Ca})_{2} \mathrm{CrRhO}_{6}, \mathrm{Sr}(\mathrm{Ca})_{2} \mathrm{MnRhO}_{6}$ and $\mathrm{Sr}$ $(\mathrm{Ca})_{2} \mathrm{FeRhO}_{6}$, respectively, would suggest $4+/ 4+$ or $3+/ 5+$ 

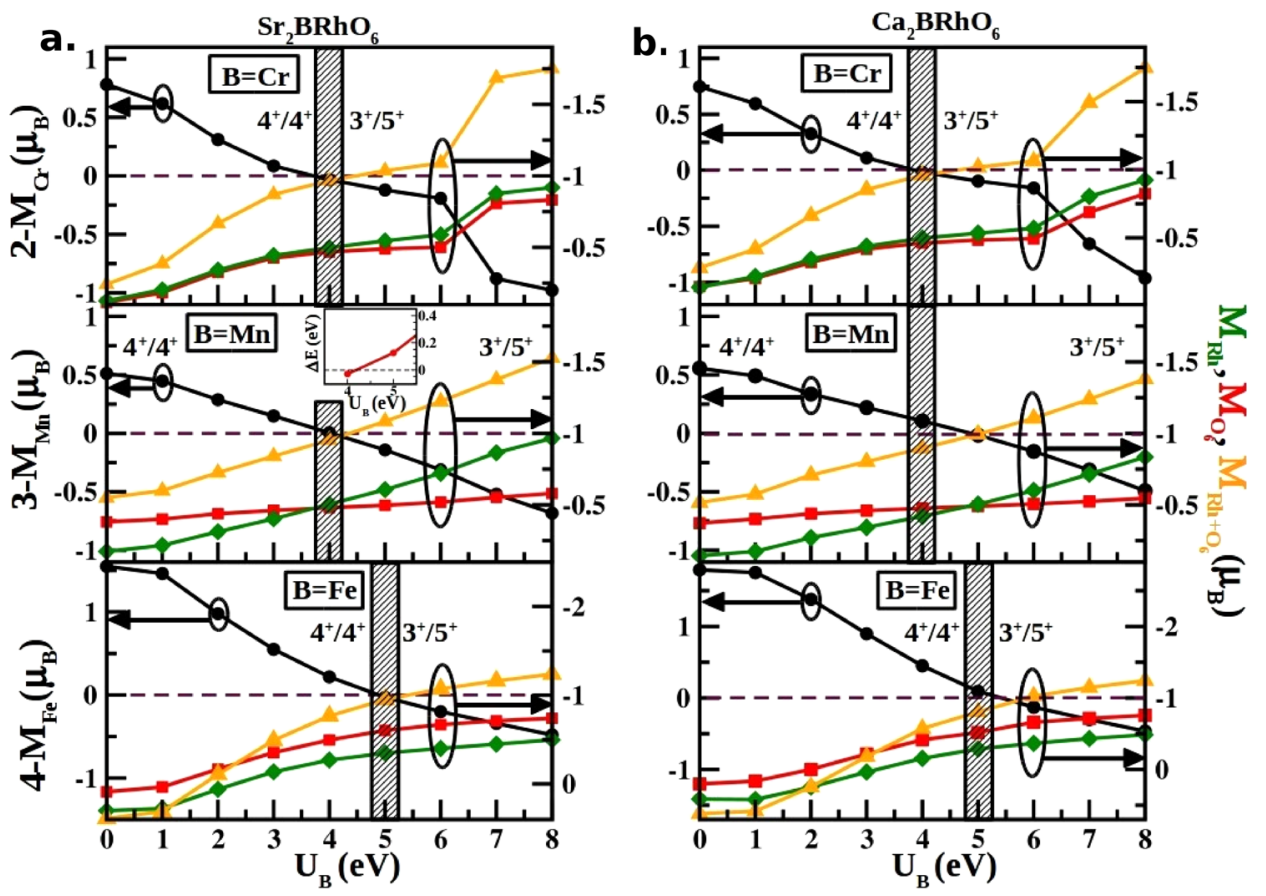

Fig. $2 U_{B}$ dependence of atom-projected magnetic moments. a Shown for $\mathrm{Sr}_{2} \mathrm{BRhO}_{6}(\mathrm{~B}=\mathrm{Cr}, \mathrm{Mn}, \mathrm{Fe})$ compounds. b Shown for $\mathrm{Ca} \mathrm{B}_{2} \mathrm{BRhO} \mathrm{O}_{6}$ $(\mathrm{B}=\mathrm{Cr}, \mathrm{Mn}, \mathrm{Fe})$ compounds. $\mathrm{B}$ site magnetic moments $\left(M_{\mathrm{B}}\right)$ are shown in left $y$-axis, while the Rh site magnetic moment $\left(M_{\mathrm{Rh}}\right)$, magnetic moments on oxygen sites $\left(M_{\mathrm{O}_{6}}\right)$, the combined moment at Rh and $\mathrm{O}$ sites $\left(M_{\mathrm{Rh}+\mathrm{O}_{6}}\right)$, all three quantities are shown in right $y$-axis. $M_{\mathrm{B}}, M_{\mathrm{Rh}}, M_{\mathrm{O}_{6}}$, $M_{\mathrm{Rh}+\mathrm{O}_{6}}$ are shown in black, green, red and yellow lines, respectively. For the moment on $\mathrm{B}$ site, plotted are the quantities, $\Delta M=2-M_{\mathrm{Cr}}, 3-$ $M_{\mathrm{Mn}}$ or $4-M_{\mathrm{Cr}}$. The change of valence at $\mathrm{B}$ site from $4+$ to $3+$ is signaled by change in $\Delta \mathrm{M}$ from a value within +1 to $0 \mu_{\mathrm{B}}$ to a value within 0 to $-1 \mu_{\mathrm{B}}$. The hatched bar in each panel signals the critical value of $U_{\mathrm{B}}$ at which such change-over happens. At this critical value of $U_{\mathrm{B}}$ $M_{\mathrm{Rh}+\mathrm{O}_{6}}$ also changes from a value less that $-1 \mu_{\mathrm{B}}$ to a value within -1 to $-2 \mu_{\mathrm{B}}$. The inset in a of the figure shows the plot of energy difference per formula unit between non-perovskite $\mathrm{C} 2 / \mathrm{m}$ symmetry and perovskite $14 / \mathrm{m}$ symmetry plotted as a function of $U_{\mathrm{B}}$ for $\mathrm{Sr}_{2} \mathrm{MnRhO}_{6}$

nominal valences for $\mathrm{B} / \mathrm{Rh}$. Following the empirical rule of ordering of $B$ and $B^{\prime}$ cations in a DP structure, ${ }^{19} 4+/ 4+$ combination may lead to disordered structure. There are, on the other hand, so far no reported examples of $\mathrm{Rh}^{5+}$ perovskites, though perovskites of neighboring elements like $\mathrm{Ru}$ and Ir, do show $5+$ valence states of $B$ cations. The only known $\mathrm{Rh}^{5+}$-based compounds, $\mathrm{Sr}_{3} \mathrm{ARhO}_{6}(\mathrm{~A}=\mathrm{Li}, \mathrm{Na})$ compounds ${ }^{20}$ are formed in $\mathrm{K}_{4} \mathrm{CdCl}_{6}$ structure type with hexagonal symmetry.

For nominal valence of $4+$ at $\mathrm{B}$ site in $\mathrm{Sr}_{2} \mathrm{BRhO}_{6}$ or $\mathrm{Ca}_{2} \mathrm{BRhO}_{6}$ which corresponds to $d^{2}, d^{3}$ and $d^{4}$ nominal occupancy of $\mathrm{Cr}, \mathrm{Mn}$ and $\mathrm{Fe}$, respectively, $M_{\mathrm{B}}$ is expected to be $1 \mu_{\mathrm{B}}<M_{\mathrm{B}}<2 \mu_{\mathrm{B}}$ for $\mathrm{B}=\mathrm{Cr}, 2 \mu_{\mathrm{B}}<M_{\mathrm{B}}<3 \mu_{\mathrm{B}}$ for $\mathrm{B}=\mathrm{Mn}$ and $3 \mu_{\mathrm{B}}<M_{\mathrm{B}}<4 \mu_{\mathrm{B}}$ for $\mathrm{B}=\mathrm{Fe}$. Decrease of nominal valence from $4+$ to $3+$ at $B$ site corresponds to $d^{3}, d^{4}$ and $d^{5}$ nominal occupancy $\mathrm{Cr}, \mathrm{Mn}$ and $\mathrm{Fe}$, respectively, which should result into magnetic moments at $\mathrm{B}$ site, $2 \mu_{\mathrm{B}}<M_{\mathrm{B}}<$ $3 \mu_{\mathrm{B}}$ for $\mathrm{B}=\mathrm{Cr}, 3 \mu_{\mathrm{B}}<M_{\mathrm{B}}<4 \mu_{\mathrm{B}}$ for $\mathrm{B}=\mathrm{Mn}$ and $4 \mu_{\mathrm{B}}<M_{\mathrm{B}}<5 \mu_{\mathrm{B}}$ for $\mathrm{B}=$ Fe. As is seen from Fig. 2, upon increasing $U_{B}$, a transition from nominal valence of $4+$ to $3+$ happens at $B$ site, at critical value of $U_{\mathrm{B}}$, estimated to be $4 \mathrm{eV}$ for $\mathrm{Cr}-\mathrm{Rh}$ and $\mathrm{Mn}-\mathrm{Rh}$ compounds, and $5 \mathrm{eV}$ for Fe-Rh compounds. At this critical value of $U_{B}$, the net moment on Rh and six $\mathrm{O}$ sites in the formula unit, $M_{R h+O_{6}}$, also makes a transition from $M_{R h+O_{6}}<1 \mu_{\mathrm{B}}$, to $1 \mu_{\mathrm{B}}<\mathrm{M}_{R h+O_{6}}<2 \mu_{\mathrm{B}}$. In order to check the influence of spin-orbit coupling (SOC), which might be important for $4 d \mathrm{Rh}$ ion, we also repeat calculations within the framework of GGA +U+SOC (see Supplementary Information, Table S3, for details of spin and orbital moments in $\mathrm{GGA}+U+\mathrm{SOC}$ calculations). The trend, as described above is found to remain valid. We thus conclude a transition of nominal 4 $+/ 4+$ valence of $B / R h$ to $3+/ 5+$ valence of $B / R h$ happens beyond critical value of $U_{B}$. As is also evident, the unusual $5+$ valence of Rh gets stabilized having a large contribution of moment from oxygen sites. Thus ideally the valence should be written as $R^{4+} \mathrm{L}$ (i.e., with a ligand hole at oxygen). The $U_{B}$-dependent change in
$\mathrm{Rh}-\mathrm{O}$ bond-length, as described in previous sub-section, further supports the change of nominal valence from $4+/ 4+$ to $3+/ 5+{ }^{20}$

The $U_{B}$-assisted stabilization of unusual $5+$ valence of Rh may be rationalized through mechanism of so-called inductive effect. ${ }^{21}$. Increase of $U_{B}$, effectively shifts the $d$ level of $B$ cation away from $p$ level of oxygen, thereby destabilizing the $O p$ states. This in turn promotes increased orbital overlap of $\mathrm{O} p$ states with $\mathrm{Rh} d$ states and facilitates the formation of ligand hole.

We also find that at the same critical $U_{B}$ value, a transition from non-perovskite structure to perovskite structure happens for $\mathrm{Sr}$ based compound. This is seen in the plot of energy difference between non-perovskite and perovskite structures, as a function of $U_{B}$, the representative case of $\mathrm{Sr}_{2} \mathrm{MnRhO}_{6}$ being presented as inset in Fig. 2a. Similar cross-over of structural stability is observed between $\mathrm{C} 2 / \mathrm{m}$ and $\mathrm{R} \overline{3}$ for $\mathrm{Sr}_{2} \mathrm{CrRhO}_{6}$ and $\mathrm{Sr}_{2} \mathrm{FeRhO}_{6}$ (see Supplementary Information Fig. $\mathrm{S} 2$ for the $U_{\mathrm{B}}$-dependent structural stability of $\mathrm{Sr}_{2} \mathrm{CrRhO}_{6}$ and $\left.\mathrm{Sr}_{2} \mathrm{FeRhO}_{6}\right)$. As mentioned before, increase of $U_{B}$ influences the Rh-O bond-length, being consistent with change in formal $\mathrm{Rh}^{4+}$ to $\mathrm{Rh}^{5+}$ valence. ${ }^{20}$ This transition from $\mathrm{Rh}(\mathrm{IV})$ to $\mathrm{Rh}(\mathrm{V})$ drives the change in structure. As noticed, this reduces the tolerance factor, e.g., from 1.03 to $1.01\left(\mathrm{Rh}^{4+} / \mathrm{Mn}^{4+}\right.$ and $\mathrm{Rh}^{5+} / \mathrm{Mn}^{3+}$ ionic radii being $0.60 / 0.53$ and $0.55 / 0.65 \AA$, respectively) ${ }^{18}$ for $\mathrm{Sr}_{2} \mathrm{MnRhO}_{6}$. The strong influence of tolerance factor on the geometry of the double-perovskites have been discussed extensively in ref. ${ }^{10}$. This shows the general trend of stabilization of non-perovskite, hexagonal symmetry-based structures for tolerance factors larger than 1, and stabilization of perovskite structures with cubic symmetry for tolerance factors about 1 or less than 1 . Our results follow this general trend.

The spin-polarized DOS of the compounds in double-perovskite space groups, $\mathrm{R} \overline{3}$ for $\mathrm{Sr}_{2} \mathrm{CrRhO}_{6}$ (SCRO), $\mathrm{Sr}_{2} \mathrm{FeRhO}_{6}$ (SFRO), $\mathrm{Ca}_{2} \mathrm{CrRhO}_{6}$ (CCRO), $\mathrm{Ca}_{2} \mathrm{MnRhO}_{6}$ (CMRO) and $\mathrm{Ca}_{2} \mathrm{FeRhO}_{6}$ (CFRO) compounds, and 14/m for $\mathrm{Sr}_{2} \mathrm{MnRhO}_{6}$ (SMRO) are shown in Fig. 3. 

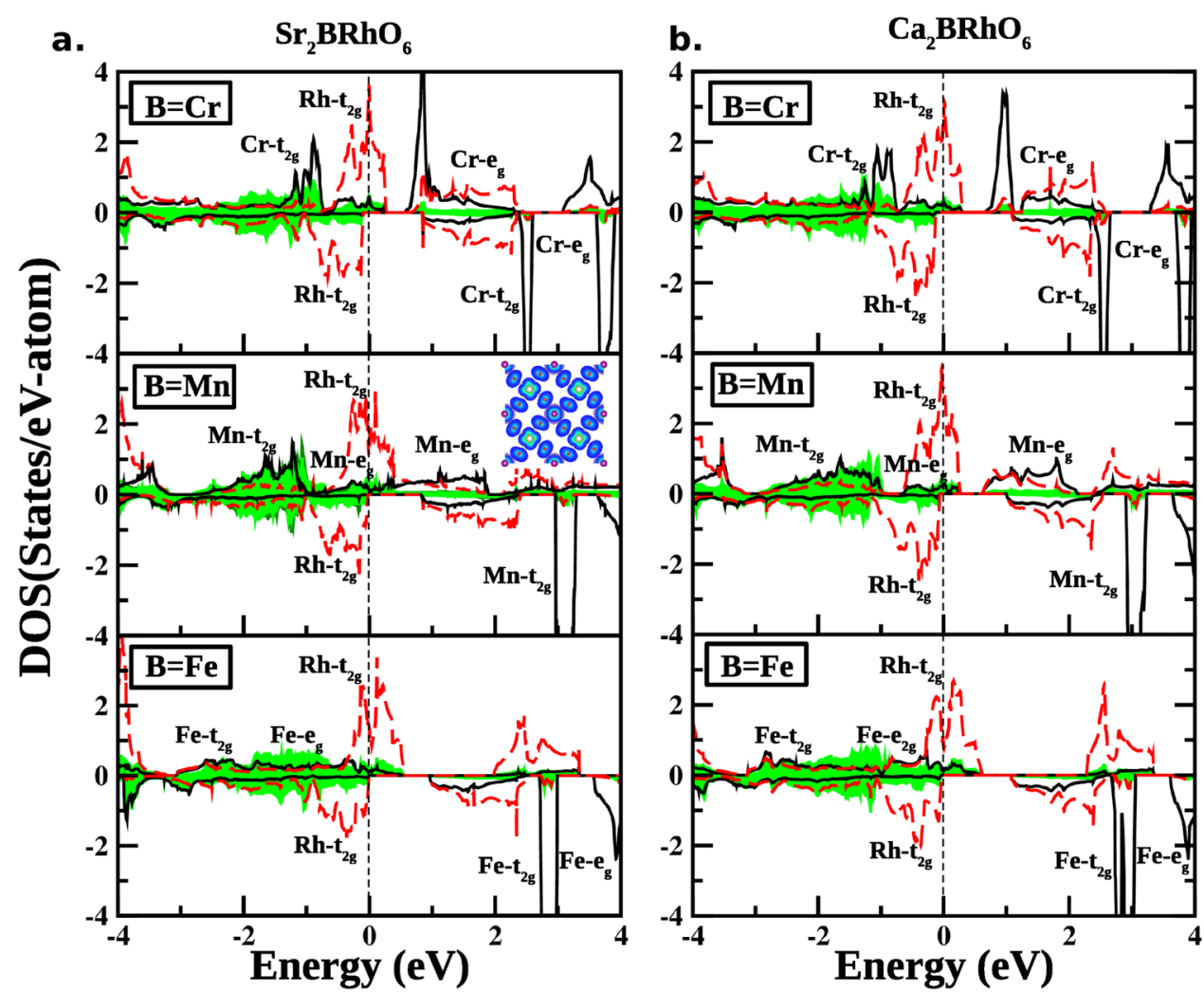

Fig. $3 \mathrm{GGA}+U\left(U_{\mathrm{B}}=5 \mathrm{eV}, U_{\mathrm{Rh}}=1 \mathrm{eV}, J_{\mathrm{H}}=0.8 \mathrm{eV}\right)$ density of states. a Shown for $\mathrm{Sr}_{2} \mathrm{BRhO}_{6}(\mathrm{~B}=\mathrm{Cr}, \mathrm{Mn}, \mathrm{Fe})$ compounds. b Shown for Ca $\mathrm{BRhO}_{6}$ $(\mathrm{B}=\mathrm{Cr}, \mathrm{Mn}, \mathrm{Fe})$ compounds. The DOS projected onto $\mathrm{B} d$ states are shown in black, solid line, $\mathrm{Rh} d$ states are shown in red, dashed lines and $\mathrm{O}$ $p$ states are shown as green, shaded area. The zero of the energy is set at GGA + $U$ Fermi energy in each case. The inset in a shows the plot of charge density calculated for the energy window from 0 to $0.5 \mathrm{eV}$. The isosurface value in the charge density plot is set at $0.015 \mathrm{e}^{-} / \AA^{3}$

We have chosen $U_{B}$ value of $5 \mathrm{eV}$ in these calculations, which is larger or equal to the critical $U_{B}$ value driving the $4+/ 4+$ to $3+/ 5+$ valence transition in the studied compounds.

A volume of literature exists on calculated $U$ values of $\mathrm{TMs}^{22-25}$ which suggest the $U$ values of $\mathrm{Cr}, \mathrm{Mn}$ and Fe atoms in oxides to be 4-5 eV. The $U$ value of a given TM atom, though depends on the environment, i.e., the particular compound under consideration. Thus, to confirm our choice, we have calculated the $U_{B}$ values of the compounds under discussion using constrained DFT calculations. ${ }^{26}$ The calculated $U_{B}$ values turned out to be 4.2 (4.3) eV for $\mathrm{Sr}$ (Ca) ${ }_{2} \mathrm{CrRhO}_{6}, 4.5$ (4.7) eV for $\mathrm{Sr}(\mathrm{Ca})_{2} \mathrm{MnRhO}_{6}$ and 5.1 (5.2) eV for $\mathrm{Sr}$ (Ca) ${ }_{2} \mathrm{FeRhO}_{6}$ justifying the uniform choice of $U_{\mathrm{B}}=5 \mathrm{eV}$ for all six compounds to be reasonable. We have also repeated our calculations within hybrid functional ${ }^{27}$ which produces results close to that obtained with $U_{\mathrm{B}}=5 \mathrm{eV}$.

Focusing on DOS plots, we find that the states close to Fermi level are dominantly of B $d$ and Rh $d$ character, which are both crystal field split into $t_{2 g}$ and $e_{g}$, and spin-split. The spin-splitting at $\mathrm{Rh}$ site is found to be oppositely oriented to that at B site in agreement with oppositely directed moments at B and Rh sites. We further find that the DOS of $\mathrm{Ca}$ and $\mathrm{Sr}$ compounds show similar features for choice of same B site element, except small decrease in band width in $\mathrm{Ca}$ compounds compared to corresponding $\mathrm{Sr}$ counterparts. We find that the $\mathrm{Rh} e_{g}$ states are empty in both spin channels in all compounds, in conformity with low spin nominal $d^{4}$ state of Rh. This makes Rh $t_{2 g}$ states completely filled in down spin channel and $\mathrm{Rh} t_{2 g}-\mathrm{O} p$ hybridized state in the up spin channel partially filled with about 1 electron. This creates a gap in down spin channel, while in up spin channel partially filled Rh $t_{2 g}-\mathrm{O} p$ states cross the Fermi energy strongly admixed with $\mathrm{Cr}_{2 g}$ states or $\mathrm{Mn} e_{g}$ states or Fe $e_{g}$ states. The inset in Fig. 3a shows the plot of charge density with an energy window set between Fermi level and $0.5 \mathrm{eV}$ above Fermi level. This shows that the unfilled low energy states have a rather large contribution from the oxygen orbitals, thereby establishing the scenario of the ligand hole formation.

\section{Magnetic ground state}

The literature of magnetic double-perovskites shows that they exhibit ferromagnetism as well as antiferromagnetism. ${ }^{7}$ In view of this we carried out total energy calculations considering the ferromagnetic (FM) alignment of B site spins, together with A-type and G-type AFM arrangement of B spins within a $2 \times 2 \times 2$ supercell of the compounds.

Comparison of the GGA $+U$ total energy of the three magnetic structures shows the FM state to the minimum energy state among the three considered magnetic structures for SCRO, SMRO, CCRO and CMRO. For choice of $U_{B}=5 \mathrm{eV}$, the FM state is found to be favored compared to A-AFM and G-AFM states by energy difference of 39.3 (49.1) meV/f.u. and 39.7 (49.4) meV/f.u., respectively, for SCRO (CCRO). SMRO and CMRO show the similar trend as SCRO and CCRO, in terms of FM state being the minimum energy state, with Ca-counterparts showing enhanced stability of FM state with respect to AFM states, compared to Sr-compounds. The calculated magnetic moments in the A-AFM and G-AFM phases, show the magnetic moment at $\mathrm{Cr} / \mathrm{Mn}$ site to be robust showing little variation between FM and AFM phases, while the the moments at $\mathrm{Rh}$ and $\mathrm{O}$ sites are found to be vanishingly small in AFM phases (see Supplementary Information, Table. S4 for calculated magnetic moments in FM and AFM phases) indicating the induced nature of moments at $\mathrm{Rh}$ and $\mathrm{O}$ sites, as will be discussed in the following. Very interestingly, moving to Fe-Rh compounds, both for Sr and Ca-counterparts, the AFM structures came out to be minimum energy states, with slight preference of 

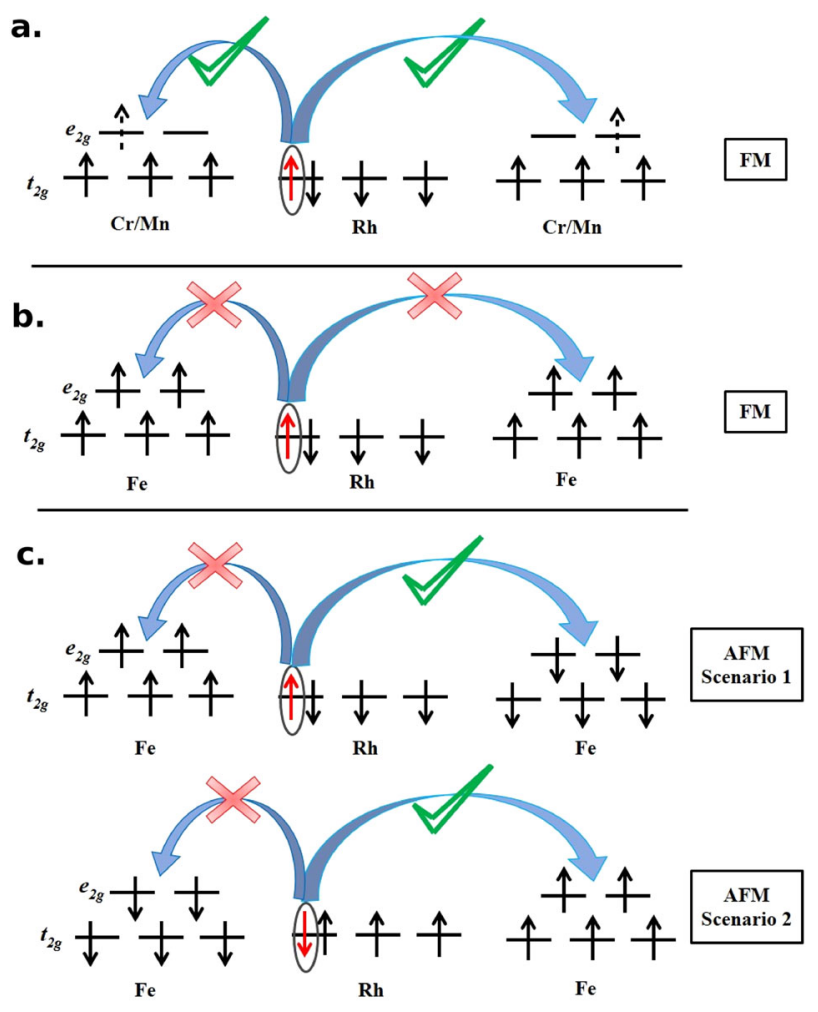

Fig. 4 The schematic represenation of allowed and dis-allowed hybridization within the hybridization-driven mechanism. a For $\mathrm{Cr}-\mathrm{Rh}$ and $\mathrm{Mn}-\mathrm{Rh}$ compounds in FM configuration of $\mathrm{Cr} / \mathrm{Mn}$ spins. $\mathbf{b}$ For Fe-Rh compounds in FM configuration of Fe spins. c For Fe-Rh compounds in AFM configuration of Fe spins

A-AFM over G-AFM, and separated by an energy difference of $109.1 \mathrm{meV} / \mathrm{f}$.u. from the FM solution for SFRO and $43.1 \mathrm{meV} / \mathrm{f}$.u. for CFRO. We find this AFM state to be metallic (see Supplementary Information, Fig. S4 for the AFM DOSs). This makes the situation as one among the rare cases of AFM metal, deviating from established wisdom of relation between magnetic order and electrical conductivity, i.e., antiferromagnets are insulators and ferromagnets are metals. There are only few examples of this exceptional situation, reported in case of compounds with lowdimensional electronic structure, ${ }^{28}$ and only one known so far in perovskite family. ${ }^{15}$

Since the above results have been obtained for a specific choice of $U_{B}$ value, the natural question to ask is that how robust is the obtained ground state upon variation of $U_{B}$ value. In order to answer this, we have carried out calculations of ground state magnetic structures, over the entire studied range of $U_{B}=1-8 \mathrm{eV}$. In all cases, the FM state turns out to be the ground state for $\mathrm{Cr}-\mathrm{Rh}$ and $\mathrm{Mn}-\mathrm{Rh}$ compounds, while the AFM state is found to be the ground state for Fe-Rh compounds (see Supplementary Information, Fig. $\mathrm{S} 3$ for $U_{B}$-dependent energy difference per f.u. between FM and AFM structures of all six compounds).

Mechanism of magnetism and model calculation of magnetic transition temperature

The fact that the moments at $\mathrm{Rh}$ and $\mathrm{O}$ site are strongly dependent on the arrangement of $\mathrm{B}$ site spins in $\mathrm{Sr}_{2} \mathrm{BRhO}_{6}$ and $\mathrm{Ca}_{2} \mathrm{BRhO}_{6}$ compounds indicates that the moment on the Rh-O network is induced by the magnetism at $\mathrm{B}$ site. This scenario was first described in the context of $\mathrm{Sr}_{2} \mathrm{FeMoO}_{6}$ in terms of hybridization driven mechanism. ${ }^{29}$ Within this mechanism, if the effective energy levels of the $4 d$ or $5 d$ TM $d-\mathrm{O} p$ fall within the exchange split energy levels of $3 d$ TM $d$, due to the hybridization between states of same spin and symmetry at the two sites, a negative spin-polarization is induced at the $4 d$ or $5 d-0$ network re-normalizing the intrinsic spin-polarization at the $4 d$ or $5 d-0$ network. Following this, in the FM configuration of $B(\mathrm{Cr} / \mathrm{Mn} / \mathrm{Fe})$ site spins, this gives rise to an intrinsic spin splitting at $3 d$ TM site and an induced, negative spin splitting at Rh network. In the AFM structure, on the other hand, where the intrinsic spins at neighboring $3 d \mathrm{TM}(\mathrm{Cr} / \mathrm{Mn} / \mathrm{Fe})$ sites are oppositely aligned, the induced spin splitting at the Rh-O network due to the effect of two neighboring $3 d$ TM sites cancel out resulting into vanishing magnetic moments at $\mathrm{Rh}$ and $\mathrm{O}$ sites. Calculated energy level diagrams of $\mathrm{B}$ site $d$ level and effective $\mathrm{Rh} t_{2 g}-\mathrm{O} p$ levels of $\mathrm{Sr}_{2} \mathrm{BRhO}_{6}$ or $\mathrm{Ca}_{2} \mathrm{BRhO}_{6}$ series, shows that effective $\mathrm{Rh}_{2 g}-\mathrm{O} p$ states appear within the spin-split energy levels of $B$ sites, further supporting the hybridization-driven mechanism of magnetism to be operative in $\mathrm{Sr}_{2} \mathrm{BRhO}_{6}$ and $\mathrm{Ca}_{2} \mathrm{BRhO}_{6}$ series.

Interesting enough, the hybridization-driven mechanism supports filling driven FM-AFM transition, which was predicted theoretically ${ }^{30}$ and verified experimentally ${ }^{31}$ for La-doped $\mathrm{Sr}_{2} \mathrm{Fe}$ $\mathrm{MoO}_{6}$. As opposed to super-exchange driven insulating AFM state found in $\mathrm{DPs}^{32}$ the AFM phase within the hybridization-driven mechanism of magnetism is metallic. The increase of filling upon moving from $\mathrm{Cr}$ or $\mathrm{Mn}$ in $\mathrm{Cr}-\mathrm{Rh}$ or $\mathrm{Mn}-\mathrm{Rh}$ compounds to $\mathrm{Fe}$ in Fe-Rh compounds, drives the Fe-Rh DPs AFM with Fe spins aligned antiparalelly in A-AFM manner with vanishing moment at $\mathrm{Rh}, \mathrm{O}$ sites, as opposed to FM ground state of $\mathrm{Cr}-\mathrm{Rh}$ or $\mathrm{Mn}-\mathrm{Rh} \mathrm{DPs}$ with parallel alignment of $B$ site moments and induced moment on Rh. As schematically shown in Fig. 4, in case of $\mathrm{Cr}-\mathrm{Rh}$ or $\mathrm{Mn}-\mathrm{Rh}$ compounds, the minority spins on Rh site, which are parallel to the majority spins on the $B$ site, can hop via the majority spin $t_{2 g}-e_{g}$ hybridization, provided all the $B$ site moments are parallel, and all the Rh site moments are antiparallel to them (cf. Fig. $4 a, b)$. In case of $\mathrm{Fe}-\mathrm{Rh}$ compounds, the $\mathrm{e}_{g}$ states in majority spin channel at $\mathrm{B}$ site gets filled, thus prohibiting $\mathrm{B}-\mathrm{Rh}$ hybridization in the majority spin channel of B site, unlike $\mathrm{Cr}-\mathrm{Rh}$ or $\mathrm{Mn}-\mathrm{Rh}$ compounds. In an AFM arrangement of $B$ site spins on the other hand (cf. Fig. 4c), both the minority and majority spin electrons at the Rh site can hybridize with the B sites in either down (Scenario 1 in the figure) or up spin (Scenario 2 in the figure) sublattices, thus maximizing the kinetic energy of hybridization. This makes Fe-Rh DPs one of the rare example of undoped DPs in AFM metallic phase.

Furthermore, it is interesting to note that the magnetism in the compounds under discussion, being governed by hybridizationdriven mechanism, as opposed to more conventional superexchange mechanism, is almost insensitive to the change in the valences from $4+/ 4+$ to $3+/ 5+$, as long as energy levels of Rh $d$ states fall within the spin-split $d$ energy levels of $B$ site. The predicted magnetic properties, thus turned out to be robust upon variation of $U_{\mathrm{B}}$ over large range, as demonstrated above.

The important ingredients in the hybridization-driven mechanism, as pointed out in refs. ${ }^{33,34}$, are (i) a large core spin (S) at B site, (ii) strong coupling between $B$ site core spin and the itinerant electron on $B^{\prime}-O$ network when it hops onto the $B$ site, inducing preferred polarization of the itinerant electron in one spin channel over other, and (iii) delocalization of the itinerant electron on the $\mathrm{B}-\mathrm{O}-\mathrm{B}^{\prime}$ network. This can be written in terms of a B and effective $B^{\prime}$ site two-sublattice double-exchange model, given by,

$H=\epsilon_{B} \sum_{i \in B, \sigma} c_{i \sigma}^{B^{\dagger} \dagger} c_{i \sigma}^{B}+\epsilon_{B^{\prime}} \sum_{i \in B^{\prime}, \sigma} c_{i \sigma}^{B^{\prime} \dagger} c_{i \sigma}^{B^{\prime}}+t \sum_{\langle i j\rangle \sigma} c_{i \sigma}^{B^{\dagger} \dagger} c_{j \sigma}^{B^{\prime}}+J \sum_{i \in B, \alpha \beta} \overrightarrow{\mathbf{s}}_{i} \cdot c_{i \sigma}^{B^{\dagger}} \vec{\sigma}_{\alpha \beta} c_{i \beta}^{B}$

where $\epsilon_{B}$ and $\epsilon_{B}$, represent onsite energies on the $B$ and $B^{\prime}$ sublattices. The third term in the Hamiltonian represents the kinetic energy of the itinerant electrons, $t$ being the effective $\mathrm{B}^{-\mathrm{B}^{\prime}}$ hopping. $c_{i \sigma}^{B \dagger}$ and $c_{i \sigma}^{B^{\prime \dagger}}$ create $B$ and $B^{\prime}$ site fermions, respectively, at the $i$-th site with spin $\sigma . \mathbf{S}_{i}^{\prime}$ s are the classical (large) core spins at B 
Table 1. $T_{\mathrm{c}} T_{\mathrm{N}}$ and exchanges $D_{\mathrm{NN}}$ and $D_{\mathrm{NNN}}$ (see text) for the model spin Hamiltonian corresponding to $\mathrm{Sr}_{2} \mathrm{BRhO}_{6}$ and $\mathrm{Ca}_{2} \mathrm{BRhO}_{6}$ compounds

\begin{tabular}{llllll}
\hline Compound & Structure & $D_{\mathrm{NN}}(\mathrm{meV})$ & $D_{\mathrm{NNN}}(\mathrm{meV})$ & $T_{\mathrm{C}}(\mathrm{K})$ & $T_{\mathrm{N}}(\mathrm{K})$ \\
\hline SCRO & Rhombohedral & -6.62 & -6.54 & 135 & - \\
SMRO & Tetragonal & -2.05 & -1.79 & 40 & - \\
SFRO & Rhombohedral & 16.6 & 18.9 & - & 145 \\
CCRO & Rhombohedral & -8.23 & -8.16 & 170 & - \\
CMRO & Rhombohedral & -6.18 & -6.71 & 135 & - \\
CFRO & Rhombohedral & 5.78 & 7.88 & - & 60 \\
\hline
\end{tabular}

site, coupled to the itinerant electrons through a coupling $J \gg t$. In the limit $J \rightarrow \infty$, the above Hamiltonian can be recast to an effective model involving only the core spin $\mathbf{S}$ of the B sites, integrating out the fermionic degrees of freedom of the itinerant electron using the procedure of self-consistent renormalization, ${ }^{34}$ given by,

$H_{\text {eff }}\{\mathbf{S}\} \approx \sum_{\langle\langle i j\rangle\rangle} D_{i j} \sqrt{\frac{1+\mathbf{S}_{i} \cdot \mathbf{S}_{j}}{2}}$

While the original Hamiltonian given by Eq. (1) contains only nearest neighbor hopping, the effective model, given by Eq. (2) contains the nearest neighbor $\left(D_{\mathrm{NN}}\right)$ as well as next nearest neighbor $\left(D_{\mathrm{NNN}}\right)$ interactions.

In the present work the Hamiltonian, $H_{\text {eff, }}$ has been used phenomenologically, and the parameters $D_{\mathrm{ij}}$ have been obtained from DFT total energies of the FM, A-AFM and G-AFM configurations. The calculated values for $D_{\mathrm{NN}}$ and $D_{\mathrm{NNN}}$ for $\mathrm{Sr}_{2} \mathrm{BRhO}_{6}$ and $\mathrm{Ca}_{2} \mathrm{BRhO}_{6}$ compounds are listed in Table 1. With the DFT inputs for $D_{\mathrm{NN}}$ and $D_{\mathrm{NNN}}$, the core spin Hamiltonian was simulated using Monte Carlo algorithm. It was confirmed that for each of the compounds same magnetic ground state was obtained from the Monte Carlo simulation as obtained from DFT. From the Monte Carlo simulation, the magnetization was calculated as a function of temperature (see Supplementary Information, Fig. S5 for plot of calculated magnetization as a function of temperature), and the FM transition temperatures, $T_{\mathrm{c}}$ was obtained for SCRO, CCRO, SMRO, and CMRO. Similarly, the staggered sublattice magnetization was calculated as a function of temperature from Monte Carlo simulation, and AFM transition temperatures, $T_{N}$ 's were obtained for SFRO and CFRO. Following the same procedure, $T_{\mathrm{c}}$ for well-known DP compound $\mathrm{Sr}_{2} \mathrm{CrMoO}_{6}$ was computed, which was found to be about $430 \mathrm{~K}$, in good agreement with experimentally measured $T_{\mathrm{C}}{ }^{5}$ thereby providing confidence in the adapted procedure. The calculated $T_{\mathrm{c}}$ and $T_{\mathrm{N}}$ values for the $\mathrm{Sr}_{2} \mathrm{BRhO}_{6}$ and $\mathrm{Ca}_{2} \mathrm{BRhO}_{6}$ compounds are listed in Table 1, which show that other than SMRO and CFRO, the magnetic transition temperatures to be above $100 \mathrm{~K}$.

\section{Predicted growth conditions}

Finally, for guiding the future synthesis of these compounds, analysis of the thermodynamic stability of the predicted Rh-based DP compounds is important. In particular, we follow the formalism outlined in refs. ${ }^{16,17}$, based on the computation of the formation enthalpies $\Delta H_{\mathrm{f}}$ of both the predicted compound and it's competing phases. The details of the analysis can be found in Supplementary Information.

This analysis provides allowed range of the deviation of the chemical potential of oxygen $\left(\mu_{\mathrm{O}}\right)$ in the compound from it's elemental-phase reference $\left(\mu_{O}^{0}\right), \Delta \mu_{O}=\mu_{O}-\mu_{O}^{0}$. The allowed range of $\Delta \mu_{\mathrm{O}}$ when recast in terms of oxygen partial pressure $\left(p_{\mathrm{O}_{2}}\right)$ and temperature $(\mathrm{T})$ provide a prediction of the growth conditions for the Rh DP compounds. Figure 5 shows the $p_{\mathrm{O}_{2}}$ vs T
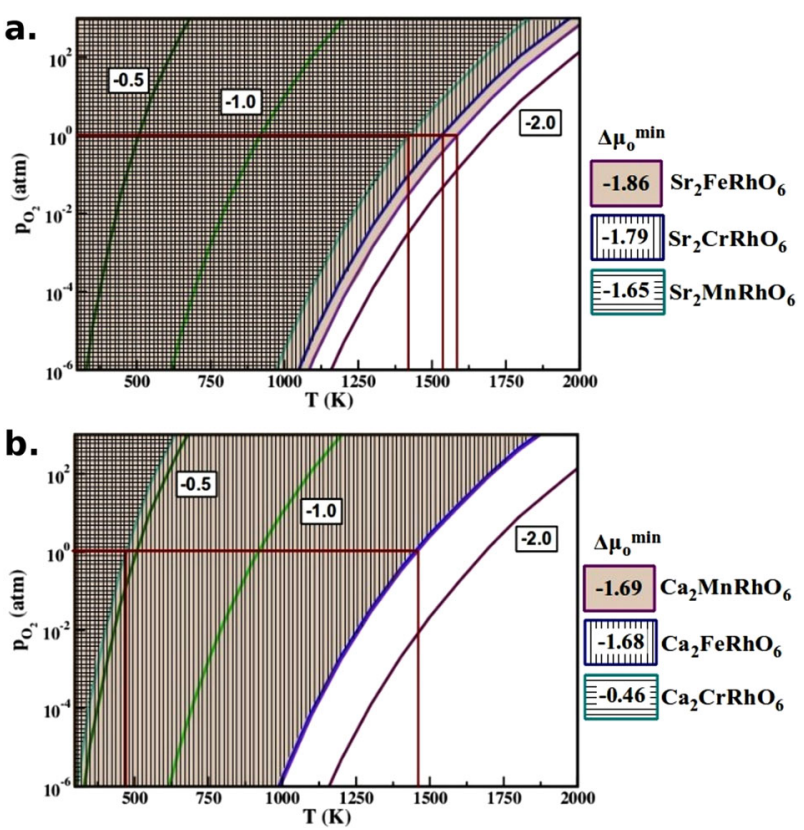

Fig. 5 Plot of oxygen partial pressure $p_{\mathrm{O}_{2}}$ vs temperature T. a Shown for $\mathrm{Sr}_{2} \mathrm{BRhO}_{6}(\mathrm{~B}=\mathrm{Cr}, \mathrm{Mn}, \mathrm{Fe})$ compounds. b Shown for $\mathrm{Ca}_{2} \mathrm{BRhO}_{6}$ $(\mathrm{B}=\mathrm{Cr}, \mathrm{Mn}, \mathrm{Fe})$ compounds. The shaded (hatched) area denotes the region corresponding to the allowed range of $\Delta \mu_{\mathrm{O}}$. The temperatures required to grow the compounds at standard atmospheric pressure are indicated by the dotted red line

plot for a range of values of $\Delta \mu_{\mathrm{O}}$. The shaded region in the figure indicates the conditions for growth of the predicted Rh-DP compounds, from which we assess that formation of $\mathrm{Sr}_{2} \mathrm{BRhO}_{6}$ and $\mathrm{Ca}_{2} \mathrm{BRhO}_{6}(\mathrm{~B}=\mathrm{Cr}, \mathrm{Mn}, \mathrm{Fe})$ compounds require high temperature synthesis for ambient pressure condition, or low pressure condition at room temperature.

\section{DISCUSSION}

Keeping in mind the cost and effort involved in synthesizing new materials, prediction of new materials with a focused need by computation is becoming increasingly popular among the researchers. In this study we consider the case of yet-tosynthesized Rh family of magnetic DPs, namely $\mathrm{Sr}(\mathrm{Ca})_{2} \mathrm{BRhO}_{6}$, where $\mathrm{B}$ is $\mathrm{Cr}$ or $\mathrm{Mn}$ or Fe. While Rh double-perovskites, having $\mathrm{Nb}$, $\mathrm{Ta}$ or Sb at B site position have been synthesized, Rh-based DPs having $3 d$ TM ions at B site position have not been reported so far. Through computer simulation, we predict the crystal structure, electronic, magnetic properties and growth conditions of these compounds, which should form an useful basis for attempts in synthesizing these compounds. We predict stabilization of unusual valence of $\mathrm{Rh}^{5+}$ through formation of ligand hole, as in case of cuprates or nickelates, ${ }^{35}$ which would be possible to verify through spectroscopic techniques if these compounds can be synthesized. Studying the electronic and magnetic structure of the compounds in predicted crystal structures, we show that while $\mathrm{Cr}-\mathrm{Rh}$ and $\mathrm{Mn}-\mathrm{Rh}$ combinations give rise to $\mathrm{FM}$, half-metallic solutions, the Fe-Rh combinations form rare example of DPs with AFM metallic properties. The microscopic origin of this exotic phase is found to be driven by hybridization-mediated mechanism, first discussed in context of $\mathrm{Sr}_{2} \mathrm{FeMoO}_{6} \cdot{ }^{29}$ The Monte Carlo simulation of the derived model Hamiltonian of the compounds show the magnetic transition temperatures to be above $100 \mathrm{~K}$ for most compounds, except $\mathrm{Sr}_{2} \mathrm{MnRhO}_{6}$ and $\mathrm{Ca}_{2} \mathrm{FeRhO}_{6}$. We believe that our study will motivate future experimental activity in terms of synthesis of these compounds with novel physical properties. 
Before closing, we discuss the possible effect of anti-site disorder, i.e., $\mathrm{Rh}$ atom sitting at position of $\mathrm{B}$ and vice-versa which is common in double-perovskites. Influence of anti-site disorder on the electronic and magnetic properties of DPs has been investigated rigorously in a number of theoretical studies. ${ }^{36-38}$ These studies suggest that in presence of moderate disorder the magnetic ground states survive with magnetic transition temperatures being largely unaffected. The anti-site disorder though are predicted to be detrimental to the strength of magnetism (e.g. the net magnetic moment or intensity of neutron peak for antiferromagnetism) as well as to the metallic or half-metallic properties. Following these findings, in presence of anti-site disorder the $T_{\mathrm{c}}$ of the $\mathrm{Cr}-\mathrm{Rh}$ and $\mathrm{Mn}-\mathrm{Rh}$ compounds are expected to remain largely unaffected, and the FM state is expected to survive with reduced magnetization and spin-polarization of carriers. Similarly, the AFM ground state of the Fe-Rh compound is expected to remain largely unaltered, except for a decrease in intensity of the magnetic neutron peaks for the antiferromagnetism. The transport behavior though is expected to change from metallic to insulating for large $(30-40 \%$, the extreme limit being $50 \%)$ antisite defect concentration. Our initial calculations also predict the crystal structure symmetry to be maintained until the extreme limit of antisite disorder, in which case DP structure converts to simple perovskite structure.

\section{METHODS}

To predict the structures of $\mathrm{Sr}_{2} \mathrm{BRhO}_{6}$ and $\mathrm{Ca}_{2} \mathrm{BRhO}_{6}(\mathrm{~B}=\mathrm{Cr}, \mathrm{Mn}, \mathrm{Fe})$ compounds, we used a genetic evolutionary algorithm which is implemented in Universal Structure Predictor: Evolutionary Xtallography (USPEX). ${ }^{39,40}$ The evolutionary algorithm for crystal structure prediction has been highly successful as a method for the discovery of minerals and materials. ${ }^{41}$ However, to the best of our knowledge, this method has not been applied to DPs, which are multi-component with four different elements, out of which two are magnetic TM ions with strong electronelectron correlation effect. We considered fixed composition mode with 10 atoms/unit cell and all the calculations are performed at zero pressure. In order to bias the search, we started with guess structures of $P 2_{1} / \mathrm{n}, 12 / \mathrm{m}$, $\mathrm{P} 4 / \mathrm{mnc}, 14 / \mathrm{m}, \mathrm{R} \overline{3}, \mathrm{Fm} \overline{3} \mathrm{~m}$ space groups, in which DPs are primarily found to form. ${ }^{10}$ The structures in the first generation were picked up randomly from the restricted space group as mentioned above. In the succeeding generations, the structures were produced employing $40 \%$ from heredity operations, $20 \%$ from lattice mutation, $10 \%$ from permutation and few structures $(30 \%)$ were produced randomly. For structural relaxations and total energy calculations, first-principle DFT-based calculations were carried out in plane wave basis as implemented in Vienna Ab initio Simulation Package ${ }^{42,43}$ which is interfaced with USPEX. We used projected augmented wave potential. ${ }^{44}$ Exchange correlation functional was chosen to be that of GGA in the implementation of Perdew-Burke-Ernzerhof. ${ }^{45}$ Additional calculations were carried out taking into account the electronic correlation effect beyond GGA at B $(\mathrm{Cr} / \mathrm{Mn} / \mathrm{Fe})$ and Rh sites through GGA $+U$ calculation ${ }^{46}$ as prescribed in Liechtenstein formulation. ${ }^{47}$ The $U$ value at $B$ site was been chosen to vary between 1 and $8 \mathrm{eV}$, keeping the $U$ value at Rh site and Hund's coupling $J_{\mathrm{H}}$ fixed at $1 \mathrm{eV}$, and at $0.8 \mathrm{eV}$, respectively. Structural relaxations were carried out stepwise from low to high precision, where at the last stage we used a full relaxation with a high threshold of energy cut off set at $500 \mathrm{eV}$ and a force convergence of $10^{-3} \mathrm{eV} / A^{0}$. In the final step, one single self consistency calculation was performed with energy cut off of $600 \mathrm{eV}$ and $8 \times 8 \times 8$ Monkhorst-Pack k-points mesh to obtain the accurate enthalpies. The crystal structure corresponding to the lowest enthalpy was considered as the chosen structure. The validity of the above described method has been checked for two well known DPs, namely $\mathrm{Sr}_{2} \mathrm{CrMoO}_{6}$ and $\mathrm{Sr}_{2} \mathrm{FeMoO}_{6}$. The method reproduced the correct space groups of the compounds, $\mathrm{Fm} \overline{3} \mathrm{~m}$ for $\mathrm{Sr}_{2} \mathrm{CrMoO}_{6}{ }^{5}$ and $14 / \mathrm{m}$ for $\mathrm{Sr}_{2} \mathrm{FeMoO}_{6}{ }^{4}$ with good agreement between measured and predicted lattice parameters, giving us confidence in our adapted method.

After determining the crystal structures, their electronic and magnetic structure were studied using DFT in plane wave basis. The site-projected magnetic moments were obtained from the difference of the projected partial charge in up and down spin channels on atom-centered spheres. The ground state magnetic structures were determined by comparing the energies of $2 \times 2 \times 2$ supercells having eight formula unit. These supercells with eight inequivalent $B$ atoms can accommodate the ferromagnetic (FM) spin alignment of B atoms as well as A-type and G-type AFM alignment of B atoms (see Supplementary Information, Fig. S1, for pictorial representation of the magnetic configurations). Upon clarification of magnetic structure, the magnetic transition temperatures were obtained from Monte Carlo study of a model spin Hamiltonian defined on a $16 \times 16 \times 16$ system containing 4096 spins. The growth conditions of the predicted compounds were obtained by calculating the formation enthalpy of the possible competing phases, as elaborated in the result section and in SI.

Data availability

All relevant data are available within the paper and its Supplementary Information file.

\section{ACKNOWLEDGEMENTS}

The authors acknowledge fruitful discussions with Pat Woodward and Ken Poeppelmeier. A.H, D.N and T.S.D acknowledge the computational support of Thematic Unit of Excellence on Computational Materials Science, funded by Nanomission of Department of Science and Technology, India. P.S acknowledges the Faculty Initiation Grant FIG 100625 of IIT Roorkee, India.

\section{AUTHOR CONTRIBUTIONS}

A.H. was responsible for carrying out the USPEX and all DFT calculations, D.N. carried out the calculations for thermodynamic stability analysis, P.S. carried out the model study. T.S-D wrote the manuscript, analyzed the results and supervised the project.

\section{ADDITIONAL INFORMATION}

Supplementary information accompanies the paper on the npj Quantum Materials website (https://doi.org/10.1038/s41535-018-0091-6).

Competing interests: The authors declare no competing interests.

Publisher's note: Springer Nature remains neutral with regard to jurisdictional claims in published maps and institutional affiliations.

\section{REFERENCES}

1. Curtarolo, S. et al. The high-throughput highway to computational materials design. Nat. Mater. 12, 191-201 (2013).

2. Stefano, S. et al. Accelerated discovery of new magnets in the Heusler alloy family. Sci. Adv. 3, e1602241 (2017).

3. Gautier, R. et al. Prediction and accelerated laboratory discovery of previously unknown 18-electron ABX compounds. Nat. Chem. 7, 308-316 (2015).

4. Kobayashi., K.-I., Kimura, T., Sawada, H., Terakura, K. \& Tokura, Y. Roomtemperature magnetoresistance in an oxide material with an ordered doubleperovskite structure. Nature 395, 677-680 (1998).

5. Patterson, F. K., Moeller, C. W. \& Ward, R. Magnetic oxides of molybdenum(V) and tungsten(V) with the ordered perovskite structure. Inorg. Chem. 2, 196-198 (1963).

6. Arulraj, A., Ramesha, K., Gopalakrishnan, J. \& Rao, C. N. R. Magnetoresistance in the double perovskite $\mathrm{Sr}_{2} \mathrm{CrMoO}_{6}$. J. Solid State Chem. 155, 233-237 (2000).

7. Philipp, J. et al. Structural and doping effects in the half-metallic double perovskite $\mathrm{A}_{2} \mathrm{CrWO}_{6}(\mathrm{~A}=\mathrm{Sr}, \mathrm{Ba}$, and Ca). Phys. Rev. B 68, 144431 (2013).

8. Kato, H., Okuda, T., Okimoto, Y. \& Tomioka, Y. Metallic ordered double-perovskite $\mathrm{Sr}_{2} \mathrm{CrReO}_{6}$ with maximal Curie temperature of $635 \mathrm{~K}$. Appl. Phys. Lett. 81, 328 (2002).

9. Krockenberger, Y. et al. $\mathrm{Sr}_{2} \mathrm{CrOsO}_{6}$ : End point of a spin-polarized metal-insulator transition by $5 d$ band filling. Phys. Rev. B 75, 020404(R) (2007).

10. Vasala, S. \& Karppinen, M. $\mathrm{A}_{2} \mathrm{~B}^{\prime} \mathrm{B}^{\prime \prime} \mathrm{O}_{6}$ perovskites: a review. Progress. Solid State Chem. 43, 1-36 (2015).

11. Jung, D.-Y. \& Demazeau, G. Structural and magnetic study on the perovskite rhodium(III) oxides, $\mathrm{Sr}_{2} \mathrm{MRh}(\mathrm{III}) \mathrm{O}_{6}(\mathrm{M}=\mathrm{Nb}$, Ta). Solid State Commun. 94, 963-967 (1995).

12. Blasse, G. New compounds with perovskite-like structures. J. Inorg. Nucl. Chem. 27, 993-1003 (1965).

13. Arribi, P. V., García-Fernández, P., Junquera, J. \& Pardo, V. Efficient thermoelectric materials using nonmagnetic double perovskites with $d^{0} / d^{6}$ band filling. Phys. Rev. B 94, 035124 (2016).

14. Coey, J. M. D. in Spin Electronics, Vol. 569 (eds Thornton, M. J. \& Ziese, M.) (Springer, Berlin Heidelberg, 2001). 
15. Komarek, A. C. et al. $\mathrm{CaCrO}_{3}$ : an anomalous antiferromagnetic metallic oxide. Phys. Rev. Lett. 101, 167204 (2008).

16. Zhang, X., Stevanovićy, V., d'Avezac, M., Lany, S. \& Zunger, A. Prediction of $A_{2} B X_{4}$ metal-chalcogenide compounds via first-principles thermodynamics. Phys. Rev. $B$ 86, 014109 (2012).

17. Osorio-Gullién, J., Lany, S., Barabash, S. V. \& Zunger, A. Magnetism without magnetic ions: percolation, exchange, and formation energies of magnetismpromoting intrinsic defects in CaO. Phys. Rev. Lett. 96, 107203 (2006).

18. Shannon, R. D. Revised effective ionic radii and systematic studies of interatomic distances in halides and chalcogenides. Acta Cryst. A 32, 751-767 (1976).

19. Anderson, M. T., Greenwood, K. B., Taylor, G. A. \& Poeppelmeier, K. R. B-cation arrangements in double perovskites. Prog. Solid State Chem. 22, 197-233 (1993).

20. Reisner, B. A. \& Stacy, A. M. $\mathrm{Sr}_{3} \mathrm{ARhO}_{6}(\mathrm{~A}=\mathrm{Li}, \mathrm{Na})$ : crystallization of a Rhodium(V) oxide from molten hydroxide. J. Am. Chem. Soc. 120, 9682-9683 (1998).

21. Etourneau, J., Portier, J. \& Ménil, F. The role of the inductive effect in solid-state chemistry: how the chemist can use it to modify both the structural and the physical properties of the materials. J. Alloys Compd. 188, 1-7 (1992).

22. Solovyev, I. V., Dederichs, P. H. \& Anisimov, V. I. Corrected atomic limit in the localdensity approximation and the electronic structure of $d$ impurities in Rb. Phys. Rev. B 50, 16861 (1994).

23. Anisimov, V. I., Elfimov, I. S., Hamada, N. \& Terakura, K. Charge-ordered insulating state of $\mathrm{Fe}_{3} \mathrm{O}_{4}$ from first-principles electronic structure calculations. Phys. Rev. $B$ 54, 4387 (1996).

24. Zhang, Z. \& Satpathy, S. Electron states, magnetism, and the Verwey transition in magnetite. Phys. Rev. B 44, 13319 (1991).

25. Sanyal, P. et al. Magnetism in $\mathrm{Sr}_{2} \mathrm{CrMoO}_{6}$ : a combined ab initio and model study. Phys. Rev. B 94, 035132 (2016).

26. Anisimov, V. I., Zaanen, J. \& Andersen, O. K. Band theory and Mott insulators: Hubbard U instead of Stoner. I. Phys. Rev. B 44, 943 (1991).

27. Paier, J., Marsman, M., Hummer, K. \& Kresse, G. Screened hybrid density functionals applied to solids. J. Chem. Phys. 124, 154709 (2006).

28. Yoshida, Y. et al. Crystal and magnetic structure of $\mathrm{Ca}_{3} \mathrm{Ru}_{2} \mathrm{O}_{7}$. Phys. Rev. B 72, 054412 (2005).

29. Sarma, D. D., Mahadevan, P., Saha-Dasgupta, T., Ray, S. \& Kumar, A. Electronic structure of $\mathrm{Sr}_{2} \mathrm{FeMoO}_{6}$. Phys. Rev. Lett. 85, 2549 (2000).

30. Sanyal, P., Das, H. \& Saha-Dasgupta, T. Evidence of kinetic-energy-driven antiferromagnetism in double perovskites: a first-principles study of La-doped $\mathrm{Sr}_{2} \mathrm{FeMoO}_{6}$. Phys. Rev. B 80, 224412 (2009).

31. Jana, $\mathrm{S}$. et al. Signature of an antiferromagnetic metallic ground state in heavily electron-doped $\mathrm{Sr}_{2} \mathrm{FeMoO}_{6}$. Phys. Rev. B 86, 054433 (2012).

32. Fang, Z., Terakura, K. \& Kanamori, J. Strong ferromagnetism and weak antiferromagnetism in double perovskites: $\mathrm{S}_{\mathrm{r}} 2 \mathrm{FeM}_{\mathrm{O}} 6(\mathrm{M}=\mathrm{Mo}, \mathrm{W}$, and Re). Phys. Rev. $B$ 63, 180407(R) (2001).

33. Chattopadhyay, A. \& Millis, A. J. Theory of transition temperature of magnetic double perovskites. Phys. Rev. B 64, 024424 (2001).

34. Sanyal, P. \& Majumdar, P. Magnetic model for the ordered double perovskites. Phys. Rev. B 80, 054411 (2009).
35. Sawatzky, G. \& Green, R. in Quantum Materials: Experiments and Theory, Vol. 6 (eds Pavarini, E., Koch, E., van den Brink, J. \& Sawatzky, G.) (Forschungszentrum Jülich, 2016). https://www.cond-mat.de/events/correl16/mauscripts/.

36. Singh, V. N. \& Majumdar, P. Antisite domains in double perovskite ferromagnets: Impact on magnetotransport and half-metallicity. Europhys. Lett. 94, 47004 (2011).

37. Sanyal, P. Antiferromagnetic metal phases in double perovskites having microscopic phase segregation due to strong antisite defect concentration. Eur. Phys. J. B 88, 294 (2015).

38. Singh, V. N. \& Majumdar, P. Antiferromagnetic order and phase coexistence in a model of antisite disordered double perovskites. Eur. Phys. J. B 83, 147 (2011).

39. Oganov, A. R. \& Glass, C. W. Crystal structure prediction using ab initio evolutionary techniques: Principles and applications. J. Chem. Phys. 124, 244704 (2006).

40. Lyakhov, A. O., Oganov, A. R. \& Valle, M. in Modern Methods of Crystal Structure Prediction (ed. Oganov, A. R.) (Wiley-VCH Verlag GmbH \& KgaA, Weinheim, 2012).

41. Oganov, A. R. \& Glass, C. W. Evolutionary crystal structure prediction as a tool in materials design. J. Phys. Condens. Matter 20, 064210 (2008).

42. Kresse, G. \& Hafner, J. Ab initio molecular dynamics for liquid metals. Phys. Rev. $B$ 47, 558(R) (1993).

43. Kresse, G. \& Furthmuller, J. Efficient iterative schemes for ab initio total-energy calculations using a plane-wave basis set. Phys. Rev. B 54, 11169 (1996).

44. Blöchl, P. E. Projector augmented-wave method. Phys. Rev. B 50, 17953 (1994).

45. Perdew, J. P., Burke, K. \& Ernzerhof, M. Generalized gradient approximation made simple. Phys. Rev. Lett. 77, 3865 (1996).

46. Anisimov, V. I., Aryasetiawan, F. \& Liechtenstein, A. I. First-principles calculations of the electronic structure and spectra of strongly correlated systems: the LDA+U method. J. Phys. Condens. Matter 9, 767-770 (1997).

47. Liechtenstein, A. I., Anisimov, V. I. \& Zaanen, J. Density functional theory and strong interactions: Orbital ordering in Mott Hubbard insulators. Phys. Rev. B 52, R5467 (1995).

(i) Open Access This article is licensed under a Creative Commons adaptation, distribution and reproduction in any medium or format, as long as you give appropriate credit to the original author(s) and the source, provide a link to the Creative Commons license, and indicate if changes were made. The images or other third party material in this article are included in the article's Creative Commons license, unless indicated otherwise in a credit line to the material. If material is not included in the article's Creative Commons license and your intended use is not permitted by statutory regulation or exceeds the permitted use, you will need to obtain permission directly from the copyright holder. To view a copy of this license, visit http://creativecommons. org/licenses/by/4.0/.

(c) The Author(s) 2018 